\title{
Intestinal handling-induced mast cell activation and inflammation in human postoperative ileus
}

\author{
F 0 The, ${ }^{1}$ R J Bennink, ${ }^{2}$ W M Ankum, ${ }^{3}$ M R Buist, ${ }^{3}$ O R C Busch, ${ }^{4}$ D J Gouma, ${ }^{4}$ S van \\ der Heide, ${ }^{5} \mathrm{R}$ M van den Wijngaard, ${ }^{1} \mathrm{~W} J$ de Jonge, ${ }^{1} \mathrm{G}$ E Boeckxstaens ${ }^{1}$
}

\begin{abstract}
${ }^{1}$ Department of
Gastroenterology and Hepatology, Academic Medical Center, Amsterdam, The Netherlands; ${ }^{2}$ Department of Nuclear Medicine, Academic Medical Center, Amsterdam, The Netherlands; ${ }^{3}$ Department of Gynaecology and Obstetrics, Academic Medical Center,

Amsterdam, The Netherlands;

${ }^{4}$ Department of Surgery,

Academic Medical Center,

Amsterdam, The Netherlands;

${ }^{5}$ Department of Allergy,

University Medical Center,

Groningen, The Netherlands
\end{abstract}

Correspondence to:

G E Boeckxstaens, Department of Gastroenterology and

Hepatology, Academic Medical

Center, Meibergdreef 9, 1105

AZ Amsterdam, The

Netherlands;

g.e.boeckxstaens@amc.uva.nl

Revised 1 May 2007

Accepted 5 June 2007

Published Online First

20 June 2007

\begin{abstract}
Background: Murine postoperative ileus results from intestinal inflammation triggered by manipulation-induced mast cell activation. As its extent depends on the degree of handling and subsequent inflammation, it is hypothesised that the faster recovery after minimal invasive surgery results from decreased mast cell activation and impaired intestinal inflammation.
\end{abstract}

Objective: To quantify mast cell activation and inflammation in patients undergoing conventional and minimal invasive surgery.

Methods: (1) Mast cell activation (ie, tryptase release) and pro-inflammatory mediator release were determined in peritoneal lavage fluid obtained at consecutive time points during open, laparoscopic and transvaginal gynaecological surgery. (2) Lymphocyte function-associated antigen-1 (LFA-1), intercellular adhesion molecule-1 (ICAM-1) and inducible nitric oxide synthase (iNOS) mRNA as well as leucocyte influx were quantified in nonhandled and handled jejunal muscle specimens collected during biliary reconstructive surgery. (3) Intestinal leucocyte influx was assessed by $99 \mathrm{mT}$ T-labelled leucocyte single photon emission computed tomography (SPECT) - computed tomography (CT) scanning before and after abdominal or vaginal hysterectomy.

Results: (1) Intestinal handling during abdominal hysterectomy resulted in an immediate release of tryptase followed by enhanced interleukin 6 (IL6) and IL8 levels. None of the mediators increased during minimal invasive surgery except for a slight increase in IL8 during laparoscopic surgery. (2) Jejunal ICAM-1 and iNOS mRNA transcription as well as leucocyte recruitment were increased after intestinal handling. (3) Leucocyte scanning $24 \mathrm{~h}$ after surgery revealed increased intestinal activity after abdominal but not after vaginal hysterectomy.

Conclusions: This study demonstrates that intestinal handling triggers mast cell activation and inflammation associated with prolonged postoperative ileus. These results may partly explain the faster recovery after minimal invasive surgery and encourage future clinical trials targeting mast cells to shorten postoperative ileus.

Postoperative ileus, characterised by a lack of coordinated motility of the entire gastrointestinal tract, leads to increased morbidity and prolonged hospitalisation, ${ }^{12}$ and represents a substantial socioeconomical burden. In the USA alone, the additional annual healthcare expenses related to this condition have been estimated to surpass US\$1 billion. ${ }^{3}$ At present, treatment is rather disappointing and limited to predominantly supportive measures. ${ }^{4}$

The introduction of minimal invasive surgical techniques (eg, laparoscopy) has speeded up postoperative recovery significantly. ${ }^{5}$ This major improvement is believed to result from minimal wound trauma and decreased release of stress hormones. ${ }^{67}$ In addition, it is becoming increasingly clear that intestinal inflammation is a key event in the pathogenesis of postoperative ileus. In rats, the degree of gut paralysis is directly proportional to the degree of intestinal handling and inflammation. ${ }^{8}$ This inflammation leads to local impaired muscle contractility and the activation of an adrenergic inhibitory neural pathway. ${ }^{10}$ Reduction of inflammatory cell influx accomplished by blockade of adhesion molecules shortens postoperative ileus ${ }^{10} 11$ and further underscores the importance of this handling-induced inflammation. As minimal invasive surgery implies limited handling of the intestine, faster recovery of motility may result from an impaired influx of inflammatory cells.

Although the exact mechanism remains unclear, we previously showed that mast cells play a pivotal role in triggering the inflammatory process. In mice, intestinal handling led to degranulation of mast cells with increased levels of mouse mast cell protease- 1 in peritoneal lavage fluid. In contrast, $\mathrm{W} / \mathrm{W}^{\mathrm{v}}$ mice, deficient in mast cells, failed to develop an intestinal muscle inflammation in response to manipulation of a bowel loop. Reconstitution of W/Wv mice with mast cells from wild-type animals restored the handling-induced inflammatory response, clearly demonstrating the importance of mast cells. Activation of resident macrophages has also been demonstrated, possibly secondary to influx of luminal bacteria during a brief episode of increased mucosal permeability. ${ }^{12}$ To what extent mast cell activation is the trigger leading to increased mucosal permeability and macrophage activation remains to be determined.

At present, the evidence supporting the importance of inflammation in humans is rather scarce, ${ }^{13}$ and data on the relationship between the degree of inflammation and clinical outcome are lacking. In addition, although we provided convincing evidence for a crucial role for mast cell degranulation in mice, no data are available in man. In the present study, therefore, we examined whether intestinal manipulation leads to mast cell degranulation and inflammation in patients undergoing conventional or minimal invasive surgery and hypothesised that clinical recovery is determined by the degree of manipulation-induced mast cell activation and inflammation.

\section{PATIENTS AND METHODS Participants}

Between December 2003 and July 2005 a total of 44 patients were enrolled in three clinical research 
protocols. The physical condition and comorbidity of potential participants were assessed during preassessment at the outpatient clinic of the department of anaesthesiology, which is part of the standard preoperative work-up. The American Society of Anesthesiologists-Physical Status classification (ASAPS) ${ }^{14} 15$ was used and comprises a scale from 1 to 6 in which 1 equals a normal healthy patient; 2 equals a patient with mild systemic disease; 3 equals a patient with severe systemic disease; 4 is a patient with severe systemic disease that is a constant threat to life; 5 equals a moribund patient who is not expected to survive without the operation; and 6 equals a declared brain-dead patient whose organs are being removed for donor purposes. ${ }^{14}$ In the present study, only patients categorised as ASA-PS 1-3 were asked to participate. In addition, patients were screened for the following exclusion criteria: intra-abdominal inflammation, preoperative radiation therapy and the use of anti-inflammatory or mast cell-stabilising drugs. Patients were included after informed consent was obtained.

\section{Study design}

The activation of mast cells and the inflammatory mediator response to intestinal handling were evaluated in protocol 1 (see below). Proinflammatory gene transcription and leucocyte recruitment within the handled intestinal muscle layer were studied in protocol 2. The occurrence of manipulation-induced leucocyte recruitment in relation to clinical recovery of bowel function and duration of hospital admission was evaluated in study protocol 3. All protocols were evaluated and approved by the Medical Ethical Review Board of the Academic Medical Center, Amsterdam, The Netherlands.

\section{Anaesthesia}

To correct for the influence of the anaesthetic technique and medication used, patients were subjected to standardised perioperative care according to our anaesthesiologist's protocol. In brief, patients were premedicated with paracetamol $1000 \mathrm{mg}$ and lorazepam $1 \mathrm{mg}$ on the evening before surgery and approximately $2 \mathrm{~h}$ before surgery. Induction of general anaesthesia was attained with propovol 2-2.5 mg/kg; fentanyl 1.5$3 \mu \mathrm{g} / \mathrm{kg}$; rocuronium $0.6 \mathrm{mg} / \mathrm{kg}$. Anaesthesia was maintained using end-tidal. $0.8 \%$ isoflurane. Postoperative pain medication was introduced after the last sampling in protocols 1 and 2 and was administered in a similar way to all patients according to our anaesthesiologist's pain protocol.

Protocol 1: mast cell activation and inflammatory mediator release during abdominal surgery

Peritoneal lavage fluid samples were collected from 18 patients, undergoing either an abdominal hysterectomy $(n=6)$, a laparoscopic resection of an adnexum $(n=6)$ or a transvaginal hysterectomy $(n=6)$. Three consecutive lavages were performed in each individual patient. The first lavage sample was collected immediately after opening of the peritoneum (basal). A second lavage sample was collected immediately after abdominal inspection and first gentle small intestinal handling (early). The final lavage sample was collected at the end of the procedure (late). As the intestine is not handled in the transvaginal hysterectomy group, only two lavages were performed in this group (ie, basal and late sampling). The systemic release of mediators in response to abdominal surgery was assessed in two blood samples (abdominal hysterectomy only), the first sample taken before induction of general anaesthesia (1 day prior to surgery) and the second sample taken at the end of the surgical procedure-that is, just before closure of the abdominal cavity. The harvested fluid and serum were used to measure the release of tryptase, tumour necrosis factor $\alpha($ TNF $\alpha$ ), interleukin $1 \beta$ (IL1 $\beta$ ), IL6 and IL8 in relation to surgical handling. The abdominal lavage was performed using $100 \mathrm{ml}$ of warm $\left(42^{\circ} \mathrm{C}\right)$ sterile $0.9 \% \mathrm{NaCl}$ solution, which was sprinkled gently onto the small intestine and its mesentery. After approximately $30 \mathrm{~s}$, peritoneal fluid (between 20 and $40 \mathrm{ml}$ ) was collected using a 22 French Foley catheter (Bard Limited, West Sussex, UK) connected to a $50 \mathrm{ml}$ catheter tip syringe.

Protocol 2: regulatory gene transcription and leucocyte influx upon intestinal handling

Jejunal muscle specimens were used to quantify regulatory gene transcription and assess the degree of inflammation. Fullthickness biopsies were obtained from patients undergoing biliary reconstructive surgery. This specific procedure was chosen because of its considerable length, providing at least sufficient time for gene transcription to occur. ${ }^{16}$ Two consecutive jejunal tissue samples were collected from 10 patients. The first specimen was collected at the beginning of the procedure and had not been touched by the surgeon until resection. The second tissue specimen, exposed to the usual handling during surgery, was collected approximately $3 \mathrm{~h}$ later. Following mucosa removal, both specimens were partitioned (5 $\mathrm{mm}^{2}$ segments) and snap-frozen in liquid nitrogen in the operating theatre, and stored at $-80^{\circ} \mathrm{C}$.

Protocol 3: abdominal leucocyte recruitment and clinical recovery Abdominal leucocyte single photon emission computed tomography (SPECT) - computed tomography (CT) scans were performed in 16 gynaecological patients to quantify the leucocyte recruitment in response to surgical handling. Eight patients undergoing an abdominal hysterectomy were compared with eight patients undergoing a vaginal hysterectomy. In each patient, a reference (basal) leucocyte scintigraphy was performed on the day of admission, $24 \mathrm{~h}$ prior to surgery. A second leucocyte scintigraphy was performed on the first postoperative day-that is, approximately $24 \mathrm{~h}$ after surgery. Clinical recovery was assessed until hospital discharge (see below for a detailed description).

\section{Methods}

Tryptase release

Tryptase concentrations were assessed at the routine clinical laboratory of the Department of Allergy, University Medical Center, Groningen, The Netherlands. The total tryptase ( $\alpha$ protryptase and $\beta$-tryptase) concentration was measured in peripheral blood and lavage fluid samples using a commercial fluoro-immunoenzyme assay (FIA) (Pharmacia Uppsala, Sweden). ${ }^{17}$

\section{Cytokine and chemokine release}

Cytokine levels were determined by cytometric bead array (BD PharMingen, San Diego, CA). In brief, $5 \mu$ l of each test sample was mixed with $5 \mu \mathrm{l}$ of mixed capture beads and $5 \mu \mathrm{l}$ of human phycoerythrin (PE) detection reagents consisting of PEconjugated anti-human IL1 $\beta$, TNF $\alpha$, IL6 and IL8. These mixtures were incubated at room temperature in the dark for $3 \mathrm{~h}$, washed and resuspended in $300 \mu \mathrm{l}$ of wash buffer. Acquisition was performed on a FACSCalibur using a 
high-throughput sampling interface (BD Biosciences, Sunnyvale, CA). Generated data were analysed using CBA software (BD PharMingen) and interpolated from corresponding standard curves generated using the mixed cytokine standard provided by the supplier. ${ }^{18} 19$

\section{Real-time reverse transcription-PCR}

Tissue specimens were homogenized and total RNA was extracted using Trizol (Invitrogen, Carlsbad, CA). The total RNA fractions were treated with DNase and reverse-transcribed using Superscript II (Invitrogen). cDNA (150 ng) was subjected to 45 cycles of lightcycler PCR (FastStartDNA Master plus SYBR Green; Roche, Basel Switzerland). The following primers were used: lymphocyte function-associated antigen-1 (LFA-1) antisense 5'-GACCCAAGT GCTCTCAGGAA-3' and sense 5'-AGGAGCACTCCACTTCAT GC-3'; intercellular adhesion molecule-1 (ICAM-1) antisense 5'CATAGAGACCCCGTTGCCTA-3' and sense 5'-GGGTAAGGT TCTTGCCCACT-3'; inducible nitric oxide synthase (iNOS) antisense 5'-TGGAAGCGGTAACAAAGGAGA-3' and sense 5'-CGATGCACAGCTGAGTGAAT-3'; and glyceraldehyde phosphate dehydrogenase (GAPDH) antisense 5'-CGACCACTTTG TCAAGCTCA-3' and sense 5'-AGGGGAGATTCAGTGTGGTG$3^{\prime}$. PCR quantification was performed by a linear regression method using the Log(fluorescence) per cycle number ${ }^{20}$ and normalised for GAPDH housekeeping gene expression. In each individual patient, the late sample value was expressed as fold increase of the early control sample value.

\section{Immunocytochemistry}

Immunocytochemical staining was performed on peritoneal cell cytospins obtained from the harvested abdominal lavage fluid. In brief, spins containing $1 \times 10^{5}$ cells were fixed in Carnoy's fixation fluid $(60 \%$ ethanol, $30 \%$ chloroform and $10 \%$ glacial acidic acid) for $30 \mathrm{~min}$ at room temperature and washed with Tris-buffered saline-Tween (TBST; 0.1\%). Non-specific binding of antibody was blocked by incubation with TBS containing $10 \%$ normal goat serum for $20 \mathrm{~min}$. Spins were incubated with anti-tryptase antibodies (mouse anti-human, 1:250) (Chemicon, Temecula, CA) for $2 \mathrm{~h}$ at room temperature. Goat anti-mouse Alexa-488 was used as secondary antibody (Molecular Probes, Invitrogen). After final washing, the spins were mounted using Vectashield mounting medium containing $5 \mu \mathrm{g} / \mathrm{ml}$ 4',6-diamidino-2-phenylindole (DAPI; Vector Laboratories, Burlingame, CA).

\section{Semi-quantitative evaluation of the degree of intestinal muscle inflammation}

Handled and non-handled jejunal muscle sections were used to assess the extent of inflammation. Leucocyte infiltration was visualised by myeloperoxidase (MPO) staining as described previously. ${ }^{11}$ After $10 \mathrm{~min}$ fixation in ice-cold acetone, transverse frozen sections $(8 \mu \mathrm{m})$ were incubated for $10 \mathrm{~min}$ with 3amino-9-ethyl carbazole (Sigma, St Louis, MO) as a substrate, disolved in sodium acetate buffer ( $\mathrm{pH}$.0) to which $0.01 \% \mathrm{H}_{2} \mathrm{O}_{2}$ was added. ${ }^{10}$

To evaluate the degree of inflammation, unmarked myeloperoxidase-stained early and late collected sections from 10 patients were scored independently by three observers (T.K., O.W. and R.vd W.). A semi-quantitative scoring scale from 0 to 4 was utilised, 0 being non-inflamed, 1 , very mildly inflamed; 2 , mildly inflamed; 3 , inflamed; and 4 , clearly inflamed. The mean of three scores, calculated for each segment, was used for statistical analysis (Wilcoxon signed rank test).
In vivo quantification of leucocyte recruitment

White blood cells (WBCs) were labelled using technetium-99m hexamethylpropyleneamine oxime ( $\left.{ }^{99 \mathrm{~m}} \mathrm{Tc}-\mathrm{HMPAO}\right)$ (Ceretec, GE Health, Eindhoven, The Netherlands) according to the consensus protocol for leucocyte labelling. ${ }^{21}$ The harvested WBC fraction of $100 \mathrm{ml}$ of blood labelled with an average of $450 \pm 10$ $\mathrm{MBq}$ of ${ }^{99 \mathrm{~m}} \mathrm{Tc}-\mathrm{HMPAO}$ was reinjected into the patient. Sixty min later, a SPECT scan of the abdomen was performed (GE Millennium Hawkeye, GE Healthcare, Den Bosch, The Netherlands) followed by a low-dose CT scan without contrast on the same gantry. CT data were used for attenuation correction and as an anatomical reference for region of interest (ROI) analysis. After data acquisition, images were processed on an Entegra workstation (GE Healthcare) using attenuationcorrected iterative reconstruction and analysed on a Hermes workstation (Nuclear Diagnostics, Stockholm, Sweden). Five consecutive abdominal SPECT slices were summed and ROIs were drawn around the small intestine and lumbar spine at the level of the ileac crest. Small bowel uptake of leucocytes was calculated as an uptake ratio expressed as a fraction of bone marrow activity, similar to analysis of leucocyte uptake assessment in inflammatory bowel disease. ${ }^{22}$ The small bowel uptake ratio determined prior to surgery was considered as basal leucocyte activity. The relative percentage difference in leucocyte activity $24 \mathrm{~h}$ after surgery was calculated using the following formula: (postoperative small bowel ratio/preoperative small bowel ratio) $\times 100 \%$.

\section{Clinical evaluation}

All patients received standard postoperative medical care according to the usual ward care protocol. Patients were visited by the research physician once daily until discharge to assess postoperative clinical recovery of bowel function (time of first flatus and time of first defecation). Patients were discharged when the following criteria were met: normal urinary tract function; spontaneous defecation; tolerance of oral fluid and solid food intake; adequate pain relief with oral analgesics; and adequate mobilisation and self-support.

\section{Statistical analysis}

Statistical analysis was performed using SPSS 12.02 software for Windows. Data were non-parametrically distributed and expressed as median values and interquartile range or median increase compared with basal values. In protocol 1, all serum samples but only vaginal hysterectomy lavage samples were analysed using a Wilcoxon signed rank test for two paired samples. For all other lavage sample series (consisting of three samples), a Friedman's two-way analysis of variance was applied. When a statistical difference was observed, a MannWhitney test was used to identify the specific sample(s) showing the significant difference. In protocol 2 , the quantitative PCR data and the semi-quantitative inflammation data were analysed using a Wilcoxon signed rank test. In protocol 3, leucocyte recruitment was analysed using the Wilcoxon signed rank test. Clinical data were analysed with a Mann-Whitney test for independent samples. P-values $<0.05$ was considered statistically significant.

\section{RESULTS}

\section{Patient demographics}

Eighteen patients participated in study protocol 1, six in each surgical intervention group. Overall mean age was 47 years, range 21-70 (transvaginal, 52 years, range 43-70; laparoscopy, 
Figure 1 (A) Peritoneal cells collected in late lavage fluid stained for tryptase (in green). Cell nuclei were counterstained with 4',6-diamidino-2-phenylindole (DAPI; blue). Individual patient tryptase concentrations during (B) open surgery, (C) laparoscopic surgery and (D) transvaginal surgery, measured in lavage fluid collected immediately after opening of the peritoneal cavity (basal), after first palpation of the small intestine during inspection of pelvic organs (early) and at the end of the procedure (late). A Wilcoxon signed rank test (vaginal samples) and Friedman two-way analysis of variance (laparoscopic and laparotomy samples) were used to determine statistical significance. Tryptase levels increased significantly in patients undergoing a laparotomy $(n=6$, $p=0.002$ ) in contrast to the laparoscopic $(n=6, p=0.5)$ or vaginal $(n=6$, $p=0.06$ ) approach. Note that no "early" lavage was performed in patients undergoing transvaginal surgery. The dotted line represents the median change in tryptase concentration of all six patients.
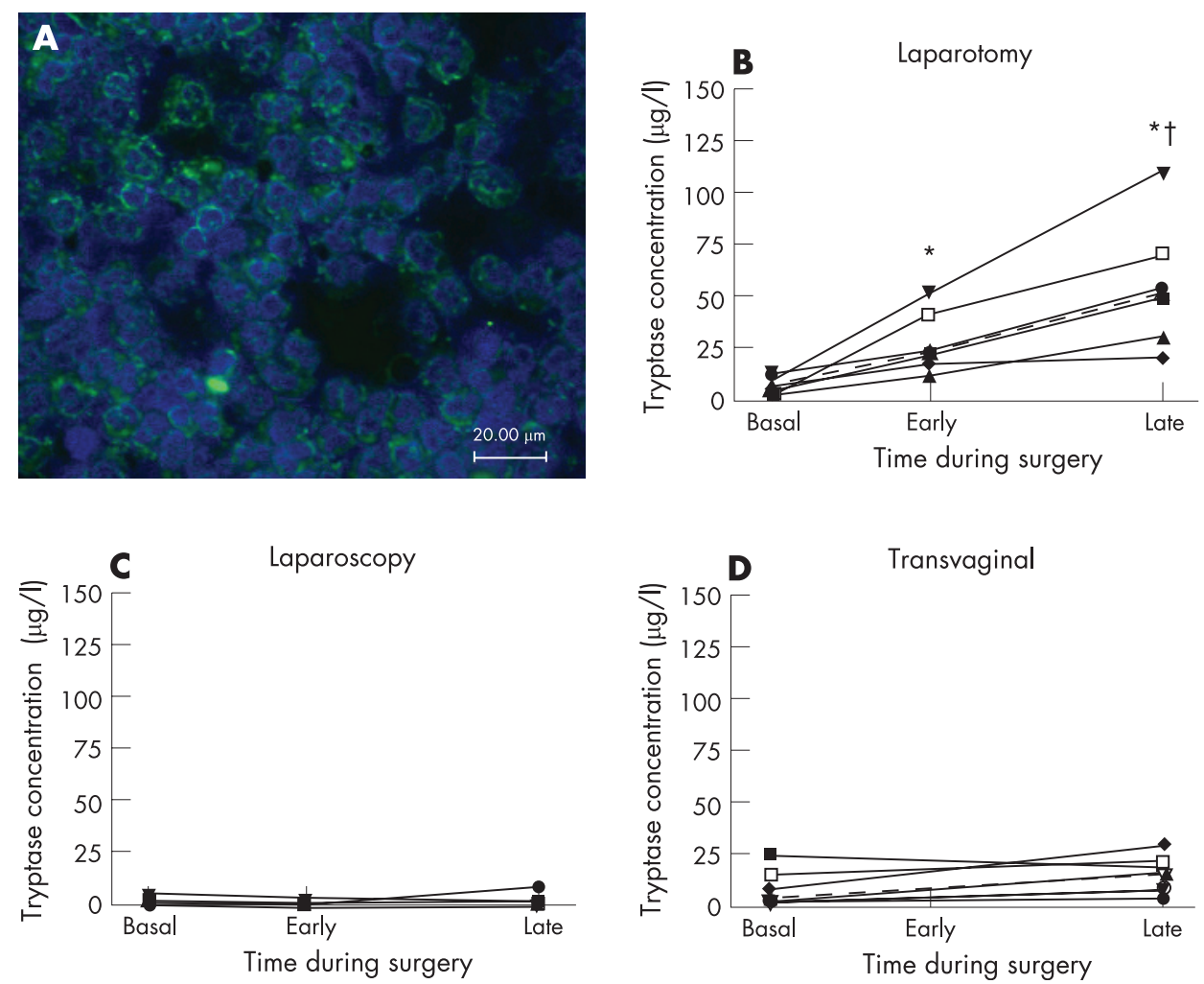

36 years, range 21-49; laparotomy, 49 years, range $44-53)$. The indications for surgery in this patient population were leiomyomata $(n=8)$, prolapse $(n=4)$ or a benign ovarian tumour $(n=6)$. In study protocol 2 , jejunal tissue samples were collected from 10 patients ( 6 male, mean age 42 years, range 3253) who underwent biliary reconstructive surgery because of iatrogenic biliary tract injury. Study protocol 3 involved 16 patients; eight patients underwent an abdominal hysterectomy (mean age 50 years, range 42-70) and eight patients underwent a vaginal hysterectomy (mean age 55 years, range 42-66). The indications for surgery were uterine leiomyomata in the abdominal hysterectomy patient group and uterine prolapse $(\mathrm{n}=4)$, leiomyomata $(\mathrm{n}=3)$ and primary dysmenorrhoea $(\mathrm{n}=1)$ in the vaginal hysterectomy group.

\section{Study protocol 1}

Mast cell activation and inflammatory response during abdominal surgery

To assess the activation of mast cells in response to intestinal handling, the expression and release of tryptase, a prestored mast cell-specific protease, ${ }^{23}$ was analysed. Peritoneal lavage fluid harvested during abdominal surgery (laparotomy) contained a distinct mast cell population, as illustrated by the number of tryptase-positive cells in fig 1A. In the basal lavage sample collected immediately after opening of the peritoneal cavity, the basal median tryptase concentration was 5.2 (interquartile range (IOR) 2.7-11.3) $\mu \mathrm{g} / \mathrm{l}$. Tryptase release was significantly increased to a median concentration of 23.1 (IOR 15.1-46.9) $\mu \mathrm{g} / \mathrm{l},(p=0.02)$ in early samples taken after gentle palpation of the small intestines, necessary to allow inspection of the pelvic organs. In the late sample taken at the end of surgery, tryptase levels had increased even further (late: median concentration 51.7 (IOR 25.8-90.2) $\mu \mathrm{g} / \mathrm{l}, \mathrm{n}=6, \mathrm{p}=0.002$ ) (fig 1B). In contrast, neither laparoscopic nor transvaginal intraperitoneal surgery ( $\mathrm{n}=6$ in both types of surgery) elicited a significant mast cell response (fig 1C,D). To evaluate possible release of mast cell mediators in the systemic circulation, we also determined preoperative and postoperative serum tryptase concentrations in the laparotomy group ( $n=6$ patients). Serum tryptase levels did not increase and remained within the normal range of $1-11.4 \mu \mathrm{g} / \mathrm{l}^{24}$ (preoperative median concentration 4.1 (IOR 2.8-7.5) $\mu \mathrm{g} / \mathrm{l}$ and postoperative 1.6 (IOR 1.3-3.5) $\mu \mathrm{g} / \mathrm{l}$, respectively).

The release of the proinflammatory cytokines TNF $\alpha$, IL1 $\beta$ and IL6, and of the chemokine IL8 was analysed in the same peritoneal lavage samples. Gentle handling of the intestine during laparotomy did not lead to an immediate increase in any of these mediators. However, at the end of the surgical procedure, IL6 and IL8 were increased significantly (table 1). In laparoscopically treated patients, intraperitoneal IL8, but not IL6, was increased, but not as profound as in the laparotomy group (table 1). On the other hand, transvaginal surgery did not affect any of the measured cytokines and chemokines. TNF $\alpha$ and IL1 $\beta$ levels did not change upon first handling or at the end of any of the types of surgery evaluated.

The serum levels of the studied inflammatory mediators remained unaltered (median increase compared with preoperatively for: TNF $\alpha, 0.0$ (IOR 0.0-0.0) pg/ml; IL1 $\beta, 0.0$ (IOR 0.035.3) pg/ml; IL6, 4.2 (IOR 0.0-11.7) pg/ml; IL8, 0.9 (IOR 0.0310.4) $\mathrm{pg} / \mathrm{ml}$.

\section{Study protocol 2}

Regulatory gene transcription upon intestinal handling Recruitment of leucocytes to the muscularis propria strongly depends on the upregulation of adhesion molecules and the synthesis of proinflammatory proteins. Therefore, ICAM-1, LFA-1 and iNOS gene expression was determined in muscle specimens collected during abdominal surgery. As the synthesis 
Table 1 Inflammatory mediator release during surgery

\begin{tabular}{lcrl}
\hline $\begin{array}{l}\text { Treatment } \\
\text { group }\end{array}$ & Laparotomy $(\mathbf{n}=\mathbf{6})$ & Laparoscopy $(\mathbf{n}=\mathbf{6})$ & Transvaginal $(\mathbf{n}=\mathbf{6})$ \\
\hline TNF $\alpha$ & $0.0(0.0$ to 3.4$)$ & $0.0(-6.6$ to 0.0$)$ & $0.0(0.0$ to 0.0$)$ \\
IL1 $\beta$ & $0.0(-1.8$ to 18.1$)$ & $-2.5(-3.3$ to 0.0$)$ & $0.2(0.0$ to 0.8$)$ \\
IL6 & $135.6(4.2 \text { to } 5130.0)^{*}$ & $6.1(1.3$ to 15.2$)$ & $1.5(-0.4$ to 4.0$)$ \\
IL8 & $114.2(32.9 \text { to } 208.7)^{* *}$ & $28.9(1.3-166.5)^{*}$ & $0.8(-2.8$ to 6.0$)$ \\
\hline
\end{tabular}

Proinflammatory mediator release was determined in lavage fluids collected immediately after opening of the peritoneal cavity (basal), after first palpation of the small intestine during inspection of pelvic organs (early) and at the end of the surgical procedure (late). Median increase of mediator concentration in the late versus the basal lavage sample collected during the indicated type of surgery $(\mathrm{pg} / \mathrm{ml}$ ) are shown with the interquartile range in parentheses. A Wilcoxon signed rank test (vaginal samples) and Friedman two-way analysis of variance (laparoscopic and laparotomy samples) were used to determine statistical significance. TNF $\alpha$ and IL1 $\beta$ did not change during surgery. IL8 increased significantly at the end of laparoscopic as well as open (laparotomy) surgery. IL6 only increased at the end of a laparotomy. None of the proinflammatory proteins increased in the transvaginal surgery group. Note that no "early" lavage was performed in patients undergoing transvaginal surgery.

${ }^{*} \mathrm{p}=0.02 ;{ }^{* *} \mathrm{p}=0.006$

IL, interleukin; TNF, tumour necrosis factor

of functional proteins requires several hours, ${ }^{16}$ mRNA quantification was used to evaluate the kinetics of these inflammatory proteins in jejunal muscle tissue. As shown in Table 2, iNOS and ICAM-1 levels were significantly increased after intestinal handling (table 2). In contrast, LFA-1 remained unchanged.

\section{Degree of intestinal muscle inflammation upon intestinal handling}

Histological evaluation of leucocyte recruitment was performed before and after surgical handling on the same tissue specimens used for gene transcription analysis. Myeloperoxidase was stained to visualise leucocyte recruitment in response to intestinal handling in transverse sections of the jejunal muscularis propria. Non-handled early samples contained only a small number of leucocytes in the muscle layer (fig 2A upper panel). In contrast, routinely handled late specimens showed a marked extravasation of inflammatory cells (fig 2A lower panel), confirmed by semi-quantitative evaluation (fig 2B). These recruited leucocytes predominantly reside in and around the vasculature of the handled intestinal muscle layers, as is illustrated in fig $2 \mathrm{~B}$. This extravasation marks the ongoing inflammatory process.

\section{Study protocol 3}

Abdominal leucocyte recruitment $24 \mathrm{~h}$ after open and minimal invasive hysterectomy

In vivo leucocyte recruitment in response to intestinal handling was investigated by $99 \mathrm{~m}$ Tc-labelled leucocyte imaging. Abdominal leucocyte influx was assessed on five consecutive leucocyte SPECT images at the level of the ileac crest and

Table 2 Quantitative gene transcription analysis

\begin{tabular}{ll}
\hline Gene & Median fold increase of gene expression \\
\hline LFA-1 & $0.9(0.3-20.0)$ \\
ICAM-1 & $3.3(1.3-139.9)^{*}$ \\
iNOS & $3.3(0.7-20.0)^{* *}$
\end{tabular}

The median fold increase of gene transcription in late (handled) versus early (nonhandled) jejunal muscle layer with the interquartile range (IOR) in parentheses $(n=10$ patients).

A Wilcoxon signed rank test was used to determine statistical differences. The relative increase was significant for iNOS (median fold increase 3.3 (IOR 0.7-20.0), $p=0.022$ ) and ICAM1 (median fold increase 3.3 (IOR 1.3-139.9), $\mathrm{p}=0.017$ ).

LFA-1, lymphocyte function-associated antigen-1; ICAM-1, intercellular adhesion molecule-1; iNOS, inducible nitric oxide synthase.

${ }^{*} \mathrm{p}=0.017 ;{ }^{* *} \mathrm{p}=0.022$. compared with that of the bone marrow. ${ }^{22}$ The change in leucocyte activity before compared with after surgery showed no increase in the vaginal hysterectomy group (median percentage activity before compared with after surgery, $91 \%$ (IOR 84-102), $\mathrm{n}=8$ ). In the abdominal hysterectomy group, however, leucocyte recruitment was significantly increased to a median of $127 \%$ of the preoperative abdominal activity ((IOR 113-148), $n=8, p=0.01$ ) (fig 3). To determine the exact anatomical location, plain CT images were made immediately after SPECT imaging. The region in which the enhanced leucocyte activity was observed coincided with small intestinal loops and its mesentery, as shown in fig 4.

\section{Clinical recovery after open and minimal invasive hysterectomy}

In conjunction with the assessed leucocyte recruitment, clinical recovery was also evaluated. Time until first flatus did not differ significantly between the two patient groups. First bowel movement and duration of hospital admission, however, were significantly prolonged after abdominal hysterectomies compared with the vaginal procedure (table 3 ).

\section{DISCUSSION}

Inflammation of the muscularis propria following surgical manipulation of the intestine is increasingly recognised to delay the recovery of gastrointestinal motility. Animal studies have revealed that prevention of this inflammatory process, by either antibodies or antisense oligonucleotides to the adhesion molecule ICAM-1, macrophage inactivation or cyclo-oxygenase-2 (COX-2) inhibition, enhances gastrointestinal transit and shortens postoperative ileus. ${ }^{11}{ }^{25-27}$ Recently, we demonstrated that mast cell activation plays an important role in this process and may be one of the first steps triggering the inflammatory response. Intestinal manipulation indeed induces the immediate activation of mast cells, leading to increased levels of the murine mast cell proteinase- 1 in the abdominal cavity. ${ }^{25}$ Three hours later, inflammatory mediators such as macrophage inflammatory protein (MIP)-2, MIP- $1 \alpha$, TNF $\alpha$ and IL6 can be detected, ${ }^{27} 28$ which in their turn enhance the expression of adhesion molecules such as ICAM- $1,{ }^{11}$ recruitment of leucocytes and inflammation of the intestine.

In the present study, we investigated whether this cascade of events also plays a role in the pathogenesis of human postoperative ileus. We found that gentle palpation of the intestines during first inspection of the pelvic organs resulted in the instantaneous intraperitoneal release of tryptase, a mast cell-specific protease, ${ }^{23}$ in patients undergoing a laparotomy. This increase in tryptase increased even further towards the end of the procedure and was accompanied by an increase in IL6 and IL8. As the latter is known to be released in response to mast cell activation and initiates leucocyte recruitment via ICAM-1, ${ }^{29}$ we also determined ICAM-1 and iNOS mRNA in intestinal tissue that was handled at the start of a surgical procedure, but was only removed approximately $3 \mathrm{~h}$ later. In addition to an upregulation of ICAM-1 and iNOS, the number of inflammatory cells was significantly increased in these late tissue samples compared with untouched specimen harvested at the beginning of the procedure. Interestingly, leucocytes were localised predominantly around blood vessels in both the serosa and the muscularis propria, partly adhering to the endothelial lining marking the ongoing recruitment and extravasation in this early stage of inflammation. Kalff et al also reported an intestinal inflammatory response during abdominal surgery in patients. ${ }^{13}$ To assess the degree of inflammation in a later stage, we also 

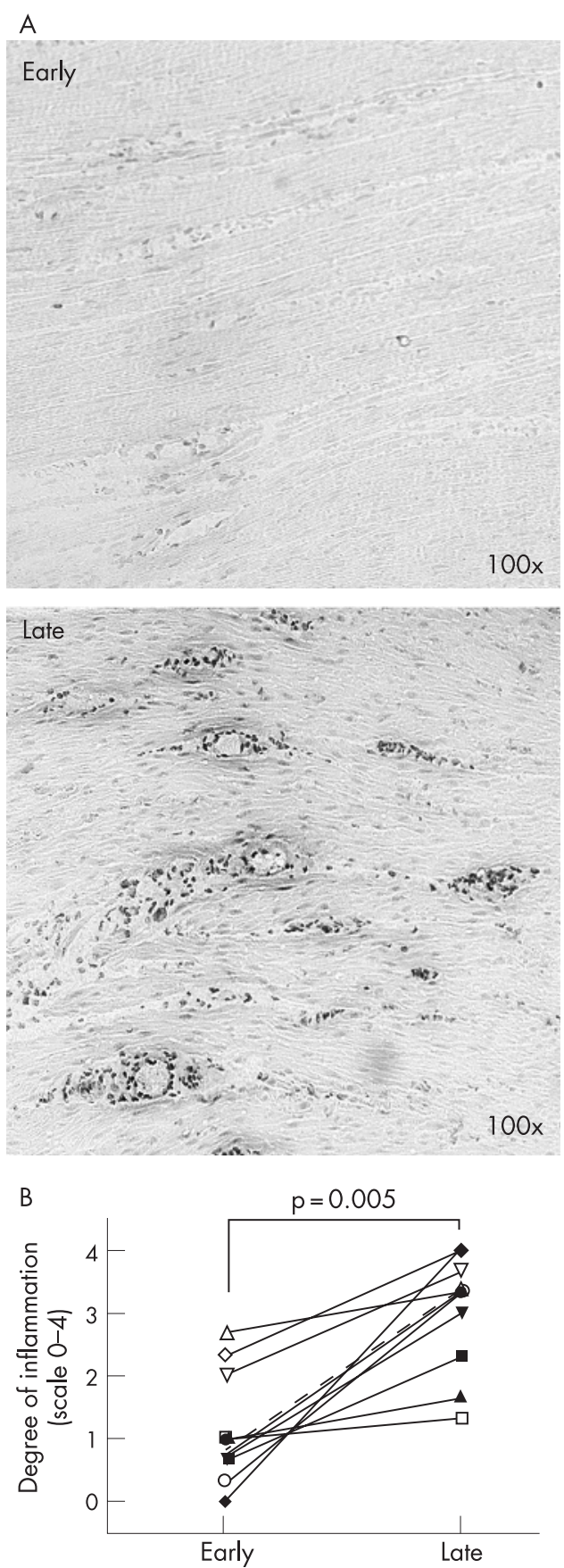

Figure 2 (A) Handling-induced leucocyte infiltration of the jejunal muscularis propria, visualised by myeloperoxidase staining in early nonhandled (upper panel) and late handled tissue segments (lower panel). Note the ongoing extravasation, illustrated by the predominant perivascular localisation of leucocytes in the handled late tissue sample. Semi-quantitative evaluation of handling-induced leucocyte recruitment (B) (scale $0=$ non-inflamed, $1=$ very mildly inflamed, $2=$ mildly inflamed, 3 = inflamed, 4 = clearly inflamed). Early non-handled (median score: 1 (IOR 1-2)) versus late handled (median score: 3 (IQR 2-4)), paired samples from $n=10$ patients, $p=0.005$ tested with a Wilcoxon signed rank test. The dotted line represents the median increase in intestinal muscle inflammation of all 10 patients.

performed ${ }^{99 \mathrm{~m}}$ Tc-labelled leukocyte SPECT scanning $24 \mathrm{~h}$ after surgery. Using this technique, we showed increased intraabdominal activity compared with the preoperative baseline scan in patients subjected to an abdominal hysterectomy. As the actual resection, performed in the pelvic region, did not

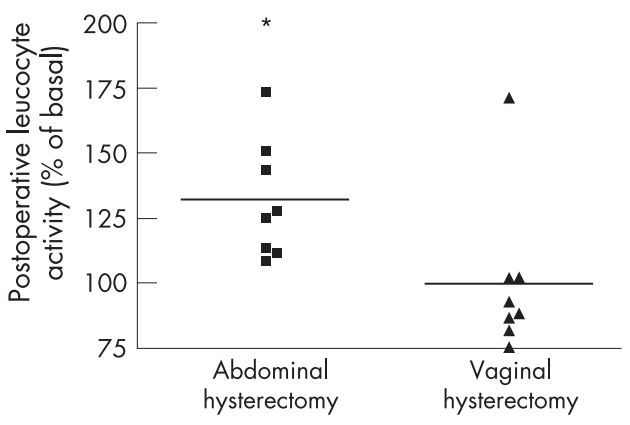

Figure 3 Quantification of postoperative leucocyte recruitment to the small intestinal region expressed as a percentage of the preoperative scan. A significant increase (Wilcoxon signed rank test) in intestinal leucocyte activity was observed after an abdominal hysterectomy (median percentage of preoperative scan, 127\% (IOR 113-148), $n=8$, $p=0.01$ ), but not after a vaginal hysterectomy (median percentage of preoperative scan, 91\% (IQR 84-102), ns, $n=8$ ).

involve any gastrointestinal organs, this observed increase in leucocyte activity cannot be explained by the primary surgical trauma. Clearly, leucocytes could reside anywhere in the abdominal cavity and may not be restricted to the intestinal wall. The additional CT scanning, however, showed that the increased leucocyte activity observed with the SPECT scans coincided with intestinal loops. When the uterus was resected transvaginally, a surgical approach that leaves the intestines largely untouched, no such increase was observed, indirectly suggesting that the intestinal inflammation is triggered by intestinal manipulation. From these data, we conclude that also in man, manipulation of the intestine during surgery leads to mast cell degranulation and a local inflammatory process, which, as in our animal model, plays an important role in postoperative hypomotility.

In rodents, the extent of gastrointestinal hypomotility or ileus is proportionally related to the degree of intestinal handling and subsequent inflammation. ${ }^{8}$ As intestinal manipulation is minimal in laparoscopic surgery, one might argue that the degree of mast cell degranulation and the subsequent inflammatory response in the intestine will be less and thus may contribute to the faster clinical recovery observed after minimal invasive surgery. To test this hypothesis, tryptase and inflammatory mediators were quantified during two minimal invasive surgical procedures-that is, laparoscopic and transvaginal hysterectomy. In contrast to gentle handling during open surgery, no mast cell degranulation or increase of IL6 was observed in the peritoneal lavage fluid. Only IL8 levels were increased, although to a lesser extent compared with laparotomy. Moreover, during transvaginal surgery, leaving the intestines largely untouched, none of the evaluated parameters increased. These findings underscore that the degree of intestinal handling to a large extent determines the degree of mast cell activation and the subsequent inflammatory response. The latter was further confirmed by the ${ }^{99 \mathrm{~m}}$ Tc-labelled leucocyte SPECT scanning $24 \mathrm{~h}$ after surgery showing increased intraabdominal activity in patients subjected to an abdominal hysterectomy but not in patients who underwent a transvaginal hysterectomy. Finally, clinical recovery in our study was significantly delayed after abdominal compared with vaginal hysterectomy, a finding in line with previous clinical studies showing faster postoperative gastrointestinal recovery after minimal invasive surgery. ${ }^{60-35}$ It should be emphasised, however, that differences in postoperative pain medication, especially opioids, may have contributed to the delay in 

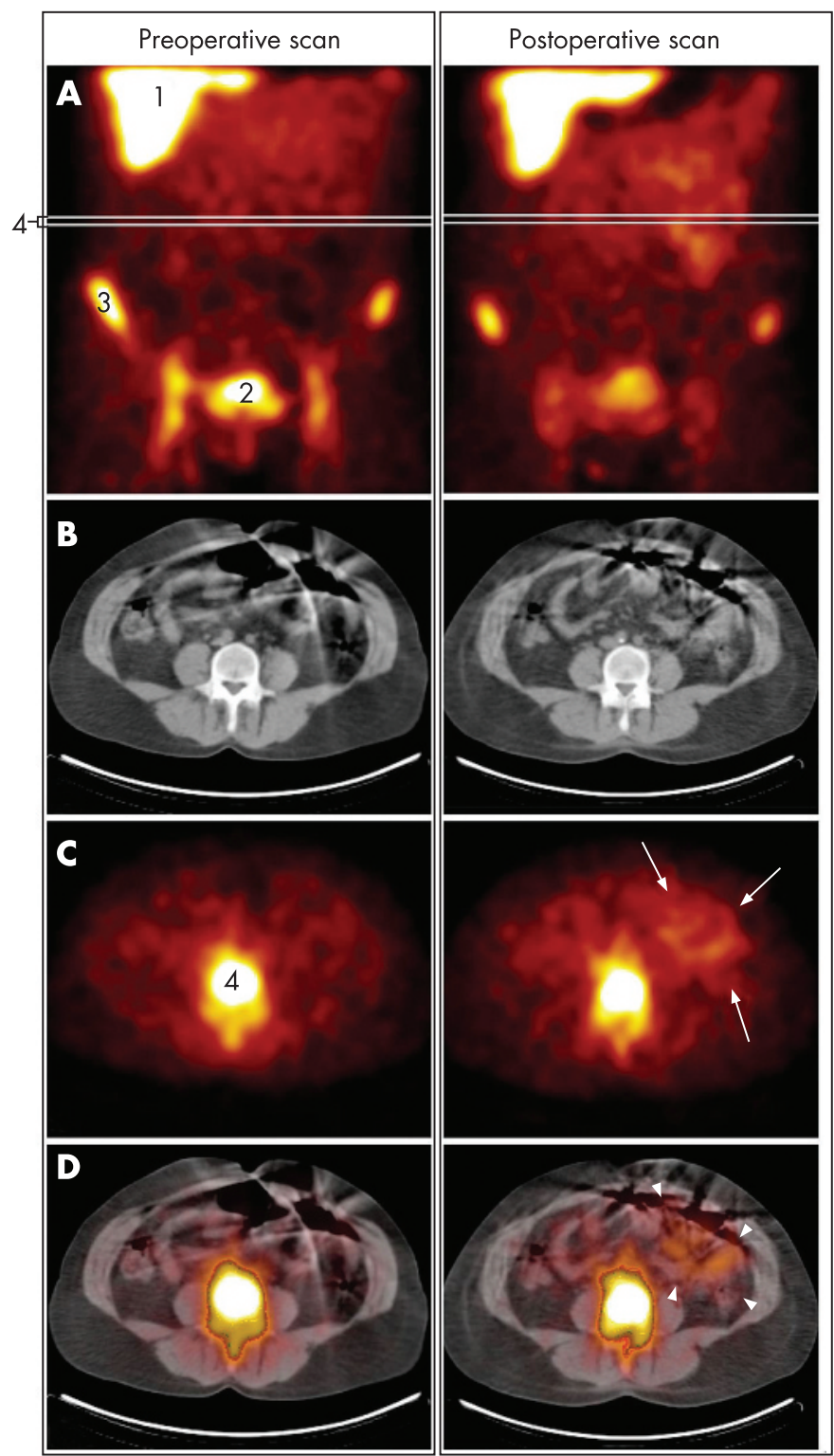

Figure 4 Representative example of leucocyte SPECT - CT imaging $24 \mathrm{~h}$ before (left column) and after (right column) an abdominal hysterectomy was performed. (A) Coronary SPECT overview slide with anatomical references: (1) liver, (2) bladder, (3) ileac spine and (4) lumbar vertebral range between which quantification was performed. (B) Transverse CT slide and (C) corresponding SPECT image at the same position in the quantification range, visualising increased leucocyte activity in the abdominal region (arrows). (D) CT and SPECT overlay showing the specific leucocyte activity in the small intestine (arrowheads).

normalisation of gastrointestinal motility. ${ }^{36}{ }^{37}$ In the current study, however, postoperative analgesia in both patient groups was provided according to a standardised postoperative pain protocol, making this explanation less likely. Therefore, the observation that delayed clinical recovery is associated with increased influx of radiolabelled leucocytes indirectly adds to the hypothesis that the degree of intestinal handling, mast cell degranulation and subsequent inflammation determines the duration of postoperative ileus.

A drawback of this study is that the study protocols were conducted in different groups of patients. Ideally, the same patient cohort should have been studied in order to understand
Table 3 Clinical evaluation of postoperative recovery

\begin{tabular}{|c|c|c|}
\hline Treatment group & Abdominal hysterectomy & Vaginal hysterectomy \\
\hline Age (years) & $50(42-70)$ & $55(42-66)$ \\
\hline ASA-PS & $2(1-3)$ & $1(1-2)$ \\
\hline Duration of surgery (min) & $182(130-298)$ & $150(113-179)$ \\
\hline $\begin{array}{l}\text { Time until first flatulence } \\
\text { (days) }\end{array}$ & $2(2-3)$ & $1(1-2)$ \\
\hline $\begin{array}{l}\text { Time until first defecation } \\
\text { (days) }\end{array}$ & $4(4-5)^{*}$ & $2(2-3)$ \\
\hline Time until discharge (days) & $8(7-8)^{* *}$ & $4(4-5)$ \\
\hline
\end{tabular}

Patient demographics and clinical recovery data from patients undergoing a vaginal hysterectomy or an abdominal hysterectomy assessed in protocol 3. To identify potential confounders in clinical parameters and to test significant difference, a MannWhitney test was performed. Age and ASA score did not differ between the two patient populations. Time till first defecation $\left({ }^{*} p=0.02\right)$ and time until discharge $\left({ }^{* *} p=0.001\right)$ were both significantly prolonged in patients undergoing an abdominal hysterectomy when compared with those undergoing a vaginal hysterectomy. All data are given as mean and (range).

ASA-PS, American Society of Anaesthesiologists-Physical Status (1 being a normal healthy patient, and 6 being a patient declared brain-dead); see the Patients and methods section for a detailed description.

better the causative association between mast cell degranulation and the subsequently observed inflammatory responses upon intestinal handling. In particular, as mRNA levels of ICAM-1 and iNOS peak only 2-24 h after stimulation, ${ }^{16}{ }^{38}$ a long-lasting surgical procedure had to be chosen in order to allow the detection of the upregulation of these inflammatory markers in response to intestinal handling. Therefore, patients undergoing biliary reconstructive surgery were selected instead.

Our current findings may have important clinical implications. First, they clearly illustrate that manipulation of the intestine should be limited whenever possible in order to reduce the release of mast cell mediators and limit postoperative intestinal inflammation. This knowledge should encourage further development of minimal invasive surgical or even endoscopic techniques to minimise intestinal handling. Secondly, if mast cell degranulation is indeed an important initial step in the pathophysiology of postoperative ileus in man, mast cells may represent an important therapeutic target. As we previously showed reduction of postoperative ileus by mast cell stabilisation in our mouse model, our current findings in humans warrant further studies evaluating the effect of a mast cell-stabilising agent in patients.

Acknowledgements: We would like to thank Professor M.P.M. Burger and Dr M. van Beurden for their support in realising the studies in the Department of Gynaecology, Professor M.W. Hollmann for anaesthesiological support and intellectual input, F. van Hemert, C. Veeris and J. de Jong for the WBC labelling, the Nuclear Medicine technicians and staff for the SPECT and CT imaging, and A. Groot for her support in the laboratory.

Funding: Supported by the Technology Foundation STW, Applied Science Division of NWO, and the Technology Program of the Ministry of Economic Affairs (NWO-STW, grant AKG 5727 to F.O.T. and R.vd W.).

Competing interests: None.

\section{REFERENCES}

1. Collins TC, Daley J, Henderson WH, et al. Risk factors for prolonged length of stay after major elective surgery. Ann Surg 1999;230:251-9.

2. Longo WE, Virgo KS, Johnson FE, et al. Risk factors for morbidity and mortality after colectomy for colon cancer. Dis Colon Rectum 2000;43:83-91.

3. Prasad M, Matthews JB. Deflating postoperative ileus. Gastroenterology 1999;117:489-92.

4. Kehlet H, Holte K. Review of postoperative ileus. Am J Surg 2001;182(5A Suppl):3S-10S

5. Schwenk W, Haase 0, Neudecker J, et al. Short term benefits for laparoscopic colorectal resection. Cochrane Database Syst Rev 2005;(3):CD003145.

6. Chen HH, Wexner SD, Iroatulam AJ, et al. Laparoscopic colectomy compares favorably with colectomy by laparotomy for reduction of postoperative ileus. Dis Colon Rectum 2000;43:61-5. 
7. Glaser F, Sannwald GA, Buhr HJ, et al. General stress response to conventional and laparoscopic cholecystectomy. Ann Surg 1995;221:372-80.

8. Kalff JC, Schraut WH, Simmons RL, et al. Surgical manipulation of the gut elicits an intestinal muscularis inflammatory response resulting in postsurgical ileus. Ann Surg 1998;228:652-63.

9. Kalff JC, Carlos TM, Schraut WH, et al. Surgically induced leukocytic infiltrates within the rat intestinal muscularis mediate postoperative ileus. Gastroenterology 1999;117:378-87.

10. de Jonge WJ, van den Wijngaard RM, The FO, et al. Postoperative ileus is maintained by intestinal immune infiltrates that activate inhibitory neural pathways in mice. Gastroenterology 2003;125:1137-47.

11. The Fo, de Jonge WJ, Bennink RJ, et al. The ICAM-1 antisense oligonucleotide ISIS3082 prevents the development of postoperative ileus in mice. Br J Pharmacol 2005; 146:252-8

12. Schwarz NT, Beer-Stolz D, Simmons RL, et al. Pathogenesis of paralytic ileus: intestinal manipulation opens a transient pathway between the intestinal lumen and the leukocytic infiltrate of the jejunal muscularis. Ann Surg 2002;235:31-40.

13. Kalff JC, Turler A, Schwarz NT, et al. Intra-abdominal activation of a local inflammatory response within the human muscularis externa during laparotomy. Ann Surg 2003;237:301-15

14. Saklad M. Grading of patients for surgical procedures. Anesthesiology 1941;2:2814.

15. American Society of Aesthesiologists. New Classification of Physical Status American Society of Aesthesiologists, Inc. Anesthesiology 1963;24:111.

16. Yan HC, Juhasz I, Pilewski J, et al. Human/severe combined immunodeficient mouse chimeras. An experimental in vivo model system to study the regulation of human endothelial cell-leukocyte adhesion molecules. J Clin Invest 1993:91:986-96.

17. Schwartz LB, Kepley C. Development of markers for human basophils and mast cells. J Allergy Clin Immunol 1994;94:1231-40.

18. Tarnok A, Hambsch J, Chen R, et al. Cytometric bead array to measure six cytokines in twenty-five microliters of serum. Clin Chem 2003;49:1000-2.

19. Chen R, Lowe L, Wilson JD, et al. Simultaneous quantification of six human cytokines in a single sample using microparticle-based flow cytometric technology. Clin Chem 1999:45:1693-4.

20. Ramakers C, Ruijter JM, Deprez RH, et al. Assumption-free analysis of quantitative real-time polymerase chain reaction (PCR) data. Neurosci Lett 2003;339:62-6.

21. Roca M, Martin-Comin J, Becker W, et al. A consensus protocol for white blood cells labelling with technetium-99m hexamethylpropylene amine oxime. International Society of Radiolabeled Blood Elements (ISORBE). Eur J Nucl Med 1998;25:797-9.

22. Weldon MJ, Masoomi AM, Britten AJ, et al. Quantification of inflammatory bowel disease activity using technetium-99m HMPAO labelled leucocyte single photon emission computerised tomography (SPECT). Gut 1995;36:243-50.
23. Hogan AD, Schwartz LB. Markers of mast cell degranulation. Methods 1997;13:4352.

24. Schwartz LB, Bradford TR, Rouse C, et al. Development of a new, more sensitive immunoassay for human tryptase: use in systemic anaphylaxis. J Clin Immunol 1994;14:190-204.

25. de Jonge WJ, The FO, van der CD, et al. Mast cell degranulation during abdominal surgery initiates postoperative ileus in mice. Gastroenterology 2004;127:535-45.

26. Schwarz NT, Kalff JC, Turler A, et al. Prostanoid production via COX-2 as a causative mechanism of rodent postoperative ileus. Gastroenterology 2001;121:1354-71.

27. de Jonge WJ, van der Zanden EP, The FO, et al. Stimulation of the vagus nerve attenuates macrophage activation by activating the Jak2-STAT3 signaling pathway. Nat Immunol 2005;6:844-51.

28. Wehner S, Behrendt FF, Lyutenski BN, et al. Inhibition of macrophage function prevents intestinal inflammation and postoperative ileus in rodents. Gut 2007:56:176-85.

29. Compton SJ, Cairns JA, Holgate ST, et al. The role of mast cell tryptase in regulating endothelial cell proliferation, cytokine release, and adhesion molecule expression: tryptase induces expression of mRNA for IL-1 beta and IL-8 and stimulates the selective release of IL-8 from human umbilical vein endothelial cells. J Immunol 1998:161:1939-46.

30. Isik-Akbay EF, Harmanli $\mathrm{OH}$, Panganamamula UR, et al. Hysterectomy in obese women: a comparison of abdominal and vaginal routes. Obstet Gynecol 2004;104:710-4.

31. Veldkamp R, Kuhry E, Hop WC, et al. Laparoscopic surgery versus open surgery for colon cancer: short-term outcomes of a randomised trial. Lancet Oncol 2005;6:47784.

32. Graber JN, Schulte WJ, Condon RE, et al. Relationship of duration of postoperative ileus to extent and site of operative dissection. Surgery 1982;92:87-92

33. Huilgol RL, Wright CM, Solomon MJ. Laparoscopic versus open ileocolic resection for Crohn's disease. J Laparoendosc Adv Surg Tech A 2004;14:61-5.

34. Bohm B, Milsom JW, Fazio WW. Postoperative intestinal motility following conventional and laparoscopic intestinal surgery. Arch Surg 1995;130:415-9.

35. Milsom JW, Hammerhofer KA, Bohm B, et al. Prospective, randomized trial comparing laparoscopic vs. conventional surgery for refractory ileocolic Crohn's disease. Dis Colon Rectum 2001;44:1-8.

36. Miedema BW, Johnson J0. Methods for decreasing postoperative gut dysmotility. Lancet Oncol 2003;4:365-72.

37. Bauer AJ, Boeckxstaens GE. Mechanisms of postoperative ileus. Neurogastroenterol Motil 2004:16(Suppl 2):54-60.

38. Yoo HS, Rutherford MS, Maheswaran SK, et al. Induction of nitric oxide production by bovine alveolar macrophages in response to Pasteurella haemolytica A1. Microb Pathog 1996;20:361-75.

\title{
Editor's quiz: GI snapshot
}

\begin{abstract}
ANSWER
From question on page 4

The sonographic characteristics of the gallbladder content excluded the diagnosis of a neoplastic process, suggesting instead a parasitic infestation. Microscopy of fresh stool sample revealed non-embryonated eggs of Fasciola hepatica. Such a trematode usually parasitises herbivorous mammals. Humans are accidentally infected by the ingestion of water or raw vegetables contaminated with the metacercaria. Human fascioliasis occurs in two steps: an acute phase, coinciding with hepatic invasion; and a chronic phase, caused by persistence of the adult form in the biliary tract, where it releases eggs. This latter situation occurred in our patient, presenting recurrent episodes of cholangitis. Anamnesis and specific ultrasound findings ${ }^{1}$ led to the correct diagnosis. The patient received triclabendazole in two oral postprandial doses of $100 \mathrm{mg}, 12 \mathrm{~h}$ apart, which resulted in eradication of the parasite.
\end{abstract}

Gut 2008;57:40. doi:10.1136/gut.2006.113928a

\section{REFERENCE}

1. Richter J, Freise S, Mull R, et al. Fascioliasis: sonographic abnormalities of the biliary tract and evolution after treatment with triclabendazole. Trop Med Int Health 1999:4:774-81. 